\title{
Michelson Interferometer Based Displacement Measurement Using Video Processing
}

\author{
V. G. BOCEKCI ${ }^{a * *}$ H. S. VAROL ${ }^{b}$ \\ ${ }^{a}$ Istanbul Technical University, Department of Electronics and Communication Engineering, \\ Engineering Faculty, 34722 Istanbul, Turkey \\ ${ }^{b}$ Marmara University, Department of Electronic-Computer Education, 34722 Istanbul, Turkey
}

\begin{abstract}
In this study, investigation of video processing method which is a new technique for precision displacement measurement is presented. The video processing method was integrated with an interferometric system and the thermal expansion results and linear thermal expansion coefficients of a heated sample metals were compared with theoretical results. Michelson interferometer setup was used in our study due to its advantages in precision displacement information of a visual output, called interferograms. In the experimental studies, signals of interferograms containing displacement information were obtained from the measurement system. The amount of displacement in the sample metals under the temperature variation due to thermal expansion was measured with the analysis program, written in Matlab/Simulink environment, with a micrometer precision. The measured displacement amounts were compared with the results of theoretical calculations. According to the theoretical values, the relative error of the values measured with video processing based method, was found found to be $0.3 \%$
\end{abstract}

DOI: 10.12693/APhysPolA.127.934

PACS: 07.60.Ly, 81.05.Bx, 07.05.Pj

\section{Introduction}

Nowadays, with the acceleration of the development of video industry, video processing technology is used for many new applications, especially in scientific, medical and engineering areas, and of course, in household applications [1-5]. Measurement applications that have an important place in the industrial and scientific studies can be carried out using the method of image and video processing [6-8]. In order to accomplish the precision displacement measurements which are important for material science, semiconductor metrology, lithography applications, the surface roughness analysis, thermal property analysis of high-tech materials and material expansion tests [9-11], by their nature, systems that require complex design are used. Interferometric displacement measurement systems are one of the commonly used systems in the precision displacement measurements [12]. Interferometric displacement systems are able to detect displacement as a result of a physical effect with a measurement accuracy of the wavelength of laser light. Michelson interferometer is one of the interferometer varieties that are recommended for this type of measurements. Michelson interferometer setup provides a visual output, called as interferograms, that can be used in determining the precision displacement by using the video processing methods. Software packages are very important due to their advantages, such as providing a good environment for applications and development of video processing. Such packages provide fast and flexible solutions for the programmer by means of functions present in their structures. One of the most important programs

*corresponding author; e-mail: gokhan.bocekci@gmail.com in which video processing applications can be carried out is MATLAB/Simulink program. In this study, the interferogram signal which contains displacement information obtained with Michelson interferometer has been analyzed using a video processing method designed on the MATLAB/Simulink program. The amount of displacement on the heated metal subjects was determined with the precision of the wavelength of the laser light. Masuda, has carried out a thermal expansion measurement of Corundum $\left(\mathrm{Al}_{2} \mathrm{O}_{3}\right)$ with a differential laser interferometer system, using He-Ne laser up to temperature of $1000 \mathrm{~K}$ [13]. Suska, has measured the linear thermal expansion coefficients of rod-shaped materials having the dimensions of $60 \times 40 \mathrm{~mm}^{2}$ and size of $100-1300 \mathrm{~mm}$ by using the laser interferometric method, between 10$40{ }^{\circ} \mathrm{C}$ [14]. Zagar, has measured the amount of heat expansion of copper samples at four different temperatures using laser interferometer separately, and then has tested the linearity of the system [15]. Demarest has described an electronic interferometric measuring system with high-resolution, high speed and reduced amount of uncertainty in laser measuring systems. In his study, he has evaluated the relationship between the structure of interferometric measuring system and measurement performance of laser displacement sensor [16]. Zhang, has measured the surface roughness and amount of displacement using an optical sensor, simultaneously. As a result of the experiments, performed on ten different surfaces, $100 \%$ reliable surface classification has been made [17]. Neubert and his colleagues have designed a high-speed dilatometer to test expansion and contraction of solid subjects. They have achieved a $0.3 \mathrm{~nm}$ resolution by using the differential interference measurement method. The induction furnace used in the system was working with induction heating and gas cooling system and was heated to $1600{ }^{\circ} \mathrm{C}$ [18]. James and his team have investi- 
gated the determination of thermal expansion coefficients of materials in the process of expansion and contraction of materials, at high temperatures of metallurgical processes, by a computer-based process modeling of thermal deformation, resulting from stress and strain of materials. They have introduced the dilatometer for measurements at high temperatures, mechanical, optical imaging and interference systems, $x$-ray diffraction methods and electrical pulse injection techniques [19]. Schödel, by taking into account the significantly growing demand for information on dimensional stability and thermal expansion properties of high-tech materials, has investigated the recent developments in elongation and thermal expansion measurements using PTB sensitive interferometers. It was found that, that the uncertainty of thermal expansion measurement is constant and being $10^{-9} \mathrm{~K}^{-1}$ or less [20]. In our study concerned with measurement of the amount of displacement of subjects under temperature variation, relative error values were at the level of $3.3 \%$ for aluminum and copper, using electronic fringe counting method [21]. The difference between the measurement system which is developed in our study and the ones from the other studies was in using of the video processing technique to detect the number of fringes in interferograms signal and its utilization for the first time to detect material's thermal elongations.

\section{The measurement system}

The displacement measurement system designed and build by us is called LETI-1 (Thermal Expansion Experimental Unit). It consists of a video camera and a displacement analysis program generated in the computer environment. The block diagram of the system is shown in Fig. 1.

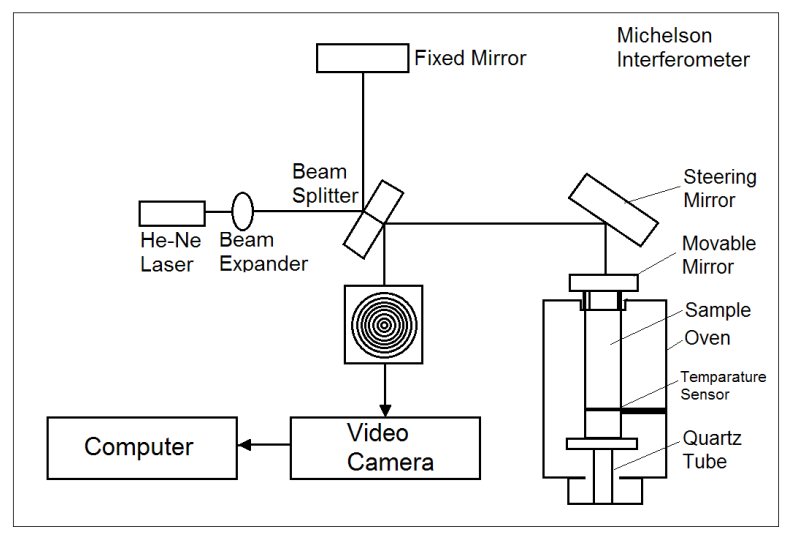

Fig. 1. Precision displacement measurement system.

LETI-1 is a test instrument used for determination of displacement of sample metals subjected to heating. LETI-1 test system consists of a temperature controlled furnace in which the furnace temperature can be set between $18{ }^{\circ} \mathrm{C}$ and $60{ }^{\circ} \mathrm{C}$ with the temperature measurement accuracy of $0.1{ }^{\circ} \mathrm{C}$. It has a chamber in which $150 \mathrm{~mm}$ long, $30 \mathrm{~mm}$ wide metal samples can be fit, a Michelson interferometer assembly, and a
$0.7 \sim 1 \mathrm{~mW}$ He-Ne laser that emites light with a wavelength of $632.8 \mathrm{~nm}$. In the Michelson interferometer fitted on LETI-1 test system, the incident laser beam from the He-Ne laser is divided into two beams by using amplitude dividing method. These light beams are reflected respectively from a fixed mirror located in the system and from a moving mirror which moves as a result of thermal expansion of sample metal in the furnace chamber, caused by temperature variation. Finally, both light beams are coupled again to get interferogram signal. In this study, in order to capture the interferogram signal and also the movement of this interferogram signal, Sony DVDDCR602e model video camera was used. During the operation, the interferogram interference signal was recorded in $736 \times 576$ video format, at 25 images per second (fps), which is the format supported by the selected video camera. The block structure of displacement analysis program created in MATLAB/Simulink environment is shown in Fig. 2.

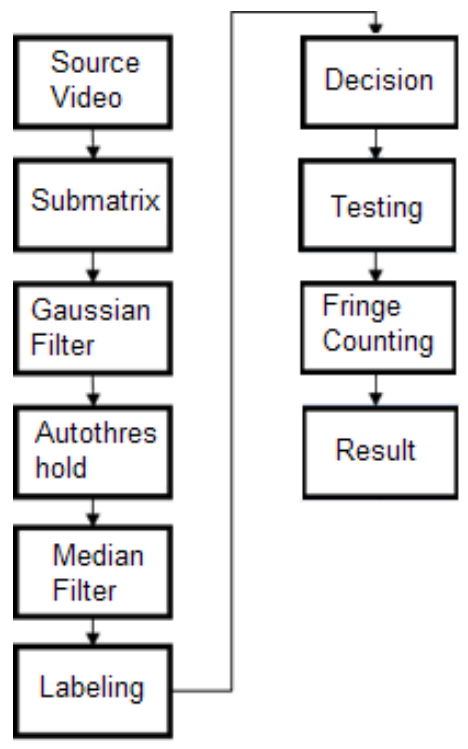

Fig. 2. Block diagram of analysis program.

Displacement analysis program consists of a source video signal block which shows the interferogram location of the video signal to be analyzed, a submatrix block which reduces the size of the interferogram signal frame, a Gaussian filter block which reduces the noise of the video signal, an autothreshold block, which converts the video signal containing the gray-scale color tones to two-color images, a median filter block which removes the salt and pepper noise in the images, a labeling block which determines the number of interference fringe signals in a single frame of video signal, and a decision block, which identifies the labeled interference fringes, a test block which determines the accuracy of the interference fringes that were discovered, a fringe counting block which determines the number of fringes in video signal and, on that basis, determines the amount of metal displacement. Number of interference fringes, determined by the counter block is transferred to the result block which will give the displacement information by using the mathematical equa- 
tion,

$$
\Delta L_{\text {measured }}=\frac{\lambda}{2} N
$$

where, $\Delta L_{\text {measured }}$ is the amount of expansion, $N$ is the number of interference fringes, $\lambda$ is the laser wavelength. Thermal expansion coefficients of aluminum and copper metals which we used as the subject material in our measurement system were $22.7 \times 10^{-6} /{ }^{\circ} \mathrm{C}$ and $20.6 \times 10^{-6} /{ }^{\circ} \mathrm{C}$, respectively. The results of experimental studies were compared with theoretical values by using the mathematical equation,

$$
\Delta L_{\text {calculated }}=\alpha L_{0} \Delta t
$$

where, $\Delta \mathrm{L}_{\text {calculated }}$ is the amount of expansion, $\alpha$ is the coefficient of linear thermal expansion, $\mathrm{L}_{0}$ is the initial length of the solid material; $\Delta t$ is the temperature range. Relative error rates of the experimental values are obtained by using the mathematical equation,

$$
\beta=\frac{\Delta L_{\text {measured }}-\Delta L_{\text {calculated }}}{\Delta L_{\text {calculated }}},
$$

where $\beta$ is the relative error rate. The coefficient of linear thermal expansion values was obtained by using the mathematical equation,

$$
\lambda \alpha=\frac{\lambda N}{2 L_{0} \Delta t},
$$

where, $\alpha$ is the coefficient of linear thermal expansion; $N$ is the number of interference fringes; $\lambda$ is the laser wavelength; $L_{0}$ is the initial length of the solid material; $\Delta t$ is the temperature range.

\section{Results and discussion}

In the measurements that were carried out between 26.5-51.5 ${ }^{\circ} \mathrm{C}$, when aluminium was used as a test material, experimental expansion values were found to be $85.40 \mu \mathrm{m}$ by using Eq. 1. In this temperature range, the theoretical expansion was determined to be $85.125 \mu \mathrm{m}$ by using Eq. 2. Relative error rate was found to be $0.33 \%$ by using Eq. 3. In the measurements that were carried out between $26.5-46.5{ }^{\circ} \mathrm{C}$, when copper was used as a test material, the experimental expansion values were found to be $62.01 \mu \mathrm{m}$ by using Eq. 1 . In this temperature range, the theoretical expansion was determined as $61.8 \mu \mathrm{m}$ by using Eq. 2. Relative error rate was found to be $0.3 \%$ by using Eq. 3. Linear thermal expansion coefficients were found by using Eq. 4. Linear thermal expansion coefficients were found to be $23.202 \times 10^{-6}$ and $20.671 \times 10^{-6}$ for aluminium and copper respectively. Based on the results obtained in this study, it is concluded that, this video processing method with a high precision of measurement results and low relative error rates, can be used as an alternative method for the precise displacement measurements.

\section{Conclusions}

In this study, video processing technique was used in order to measure the thermal expansion of metals. In electronic thermal expansion measurements, the noise effects appearing on the interferogram signals are removed using hardware solutions but in our study, the noise effects are removed by using the designed software and this software provides a decrease in system costs. The results obtained with video processing technique are compared with theoretical values. The obtained relative error value is found to be $0.3 \%$. While the same measurement is performed by using fringe counting method, the relative error value is found to be $3.3 \%$. Based on our results, it is concluded that video processing technique, which we recommend as a new method for precision displacement measurement, can be used as a suitable and precise method for the measurement of displacement.

\section{References}

[1] A. Bovik, Handbook of Image and Video Processing, Academic Press, San Diego, (2000).

[2] G. Marbach, M. Loepfe, T. Brupbacher, Fire Safety J. 41, 285 (2006).

[3] M. Bramberger, A. Doblander, A. Maier, B. Rinner, IEEE Computer Society 39, 68 (2006).

[4] H. Müller, N. Michoux, D. Bandon, A. Geissbuhler, Int. J. Med. Inform. 73, 1 (2004).

[5] F. Zhu, J. Ning, Y. Ren, J. Peng, Elektron. Elektrotech. 18, 91 (2012).

[6] F. Chen, G.M. Brown, M. Son, Opt. Eng. 39, 10 (2000).

[7] P. Nakroshis, M. Amoroso, J. Legere, C. Smith, Am. J. Phys. 71, 568 (2003).

[8] D.J. White, W.A. Take, M.D. Bolton, Géotechnique 53, 619 (2003).

[9] H. Bosse, G. Wilkening, Meas. Sci. Technol. 16 , 2155 (2005).

[10] H.H. Solak, D. He, W. Li, F. Cerrina, J. Vac. Sci. Technol. B 17, 3052 (1999).

[11] G. Bianchini, M. Barucci, T. Rosso, E. Pasca, G. Ventura, Meas. Sci. Technol. 17, 689 (2006).

[12] D. Lin, X. Jiang, F. Xie, W. Zhang, L. Zhang, I. Bennion, Optics Express 12, 5729 (2004).

[13] K. Masuda, D. Erskine, O.L. Anderson, American Meniralogist 85, 279 (2000).

[14] J. Suska, J. Tschirnich, Meas. Sci. Technol. 10, 211 (1999).

[15] B. Zagar, IEEE Transaction on Instrumentation and Measurement 43, 332 (1994).

[16] F.C. Demarest, Meas. Sci. Technol. 9, 1024 (1998).

[17] K. Zhang, C. Butler, Y. Qingping, Y. Lu, IEEE Transaction on Instrumentation and Measurement 46, 899 (1997).

[18] H. Neubert, E. Bindl, M. Mehnert, H. Rädel, C. Linseis, Meas. Sci. Technol. 20, 101 (2009).

[19] J.D. James, J.A. Spittle, S.G.R. Brown, R.W. Evans, Meas. Sci. Technol. 12, 153 (2001).

[20] R. Schödel, Meas. Sci. Technol. 19, 1 (2008).

[21] V.G. Böcekçi, H.S. Varol, Acta Phys. Pol. A 121, 181 (2012). 